\title{
Advanced System Interrogation Based on Active Bifurcation Morphing
}

\author{
Shih-Hsun Yin* and Bogdan I. Epureanu ${ }^{\dagger}$ \\ Department of Mechanical Engineering, University of Michigan \\ 2350 Hayward Street, Ann Arbor, MI 48109-2125
}

\begin{abstract}
This paper demonstrates a novel system interrogation method based on observing the morphing of bifurcations and the post-bifurcation dynamics through both experimental and numerical methods. A sensing cantilever beam is built with PZT patches symmetrically bonded to both sides of its root. A desired bifurcation in the dynamics of the beam can be induced by applying a nonlinear feedback excitation to the beam. The nonlinear feedback excitation requires the active measurement of the dynamics and a feedback loop, and is generated by applying the voltage (resulting from a nonlinear feedback) to the piezo electrode. Also, a finite element model is used to design the nonlinear feedback excitation and predict the response of the sensing beam. Numerical simulations and experiments are performed and compared to demonstrate the effectiveness and high level of sensitivity of the novel approach to detect very small amounts of mass added at the tip of the beam.
\end{abstract}

\section{Nomenclature}

C Damping matrix in a finite element model

F Forcing vector

K Stiffness matrix in a finite element model

M Mass matrix in a finite element model

$\mathrm{X} \quad$ State space vector in a finite element model

a Linear gain

$c \quad$ Damping in a one-degree-of-freedom model

$k \quad$ Stiffness in a one-degree-of-freedom model

$m$ Mass in a one-degree-of-freedom model

$p_{i} \quad$ Parameters of the nonlinear controller

$v \quad$ Velocity at the tip of the sensing beam

$\Omega \quad$ Frequency of the harmonic component of the excitation

$\gamma, \delta$ Nonlinear gains

$\xi \quad$ Damping ratio

\section{Introduction}

Vibration-based techniques are commonly used for system interrogation in applications such as structural health monitoring. ${ }^{1,2}$ Such methods monitor changes in vibratory characteristics of a structure which reflect

${ }^{*} \mathrm{PhD}$ candidate, Email: sy in@umich.edu

${ }^{\dagger}$ Assistant Professor, Member AIAA, Email: epureanu@umich.edu 


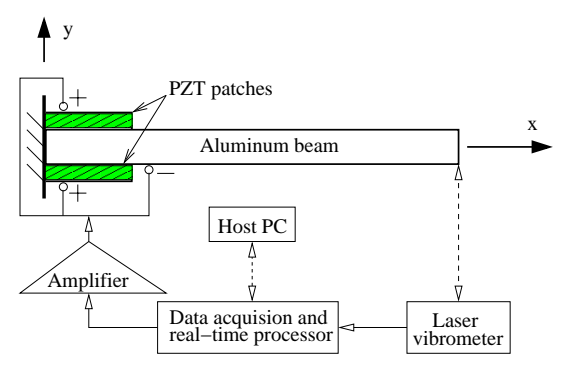

Figure 1. Schematic plot of the experimental setup and the photo of the sensing beam.

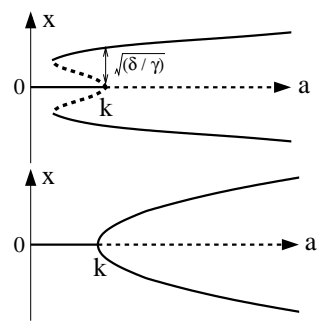

Figure 2. Fixed points of an oscillator forced by nonlinear feedback excitation and undergoing a subcritical (top) and tions.

damage. Several of these techniques use subspace identification and updating, ${ }^{3-6}$ wavelet analyses, ${ }^{7,8}$ and Ritz vectors. ${ }^{9,10}$ Although there have been numerous studies showing that changes in observed linear features can be used to detect the presence of damage, the low sensitivity of these features (to damage) limits the applicability of such methods. To increase sensitivity to parameter variations indicative of damage, the concept of sensitivity enhancing control ${ }^{11-13}$ and designed impedance techniques ${ }^{14-16}$ were proposed through linear feedback control applied to a structure. Such approaches reduce modal frequencies to enhance their sensitivity to changes in stiffness. Other studies have exploited nonlinearities to enhance sensitivity. For example, linear systems subjected to chaotic excitation ${ }^{17-21}$ and (nonlinear or) chaotic systems (with or without excitation $)^{22-24}$ have been explored to show that the use of nonlinearity holds a great potential for damage detection. Furthermore, enhancing nonlinearity within linear or weakly nonlinear systems by means of nonlinear feedback excitation has been demonstrated computationally to provide significant advantages such as increased sensitivity. ${ }^{25,26}$

In the present work, we apply a novel detection method for identifying small parametric changes in a smart structure. The method is based on observing the morphing of bifurcations and the dynamics in the post-bifurcation regime. For most structures, dissipative mechanisms balance the external excitation such that the dynamics evolves onto an invariant manifold of the state space (the attractor). Examples of attractors of the dynamics of a structure are stable fixed points, stable limit cycles, and strange attractors (for chaotic systems). A qualitative change in the dynamics (referred to as a bifurcation) may happen as parameters are varied. For example, fixed points or limit cycles can be destroyed or created, or their stability can change when bifurcations occur. The key idea of the method herein is to actively change the original stability of a fixed point (equilibrium state) or the dynamic response of a system (structure) and create a new stable fixed point, a limit cycle or a more complex dynamic response by applying a nonlinear feedback excitation to the structure. The controller parameters (gains) of the nonlinear feedback excitation are manipulated to interrogate the system by identifying and characterizing bifurcation points. The main concept and key theoretical investigations of this approach have been presented by the authors. ${ }^{27}$

In contrast, the goal of this paper is to test and validate the proposed method in an experimental way. Therefore, a smart structure composed of a cantilever beam in which two PZT (lead zirconate titanate) patches are symmetrically bonded to both sides of the root of the beam is built as shown inFig. 1. Piezoelectric actuators and sensors are currently used as elements of intelligent structures ${ }^{28}$ and are widely exploited for active vibration suppression and structural health monitoring in smart structures. ${ }^{29-32}$ In this work, a complex dynamics of the beam can be induced by applying a designed nonlinear feedback excitation to the beam. First, the dynamics at the tip of the beam is measured using a laser vibrometer, and the measured signal is used in a real-time processor where a designed nonlinear control circuit is uploaded from a host computer. Finally, the output voltage from the real-time processor is applied to the PZT patches to excite the beam. The actual bifurcation point of the dynamics can be determined by sweeping the values of the gains in the nonlinear control circuit and recording the values at which bifurcations occur. Also, a finite 


\begin{tabular}{|l|c|c|}
\hline & Aluminum beam & PZT patch \\
\hline Length $(\mathrm{mm})$ & 280 & 60 \\
\hline Width $(\mathrm{mm})$ & 15 & 15 \\
\hline Thickness $(\mathrm{mm})$ & 1.27 & 1 \\
\hline Young's modulus $(\mathrm{GPa})$ & 68.9 & 62 \\
\hline Density $\left(\mathrm{kg} / \mathrm{m}^{3}\right)$ & 2660 & 7800 \\
\hline Poisson's ratio & 0.33 & 0.31 \\
\hline Piezoelectric constant $\left(10^{-9} \mathrm{~mm} / \mathrm{V}\right)$ & - & -300 \\
\hline
\end{tabular}

Table 1. The dimension and properties of the sensing beam.

element model of the sensing beam is used to design the closed loop controller and to predict the bifurcation point and the post-bifurcation dynamics. Results from numerical simulations and experiments are compared to demonstrate the novel approach and to evaluate its effectiveness and robustness.

\section{System interrogation methodology}

In this work, the proposed method is based on observing the onset of bifurcations and the emerging dynamics in the post-bifurcation regime. The controller parameters for the nonlinear feedback excitation are manipulated to interrogate the system by identifying the bifurcation point. Consider, for clarity, the very simple example of a single degree-of-freedom oscillator forced by a specific feedback excitation. The governing equation for this simple oscillator can be expressed as

$$
m \ddot{x}+c \dot{x}+k x=a x-\delta x^{3},
$$

where $m, c$, and $k$ are mass, damping, and stiffness parameters, $a$ and $\delta$ are the linear and nonlinear controller parameters. All these parameters are assumed positive. A supercritical pitchfork bifurcation occurs when $a$ equals $k$, as shown in Fig. 2. Thus, the stiffness $k$ could be directly interrogated by varying the value of $a$ and observing the onset of the bifurcation for this special case. Hence, the change in stiffness due to damage can be detected by distinguishing between the bifurcation points for a healthy and a damaged system. Also, the type of bifurcation and the dynamics in the post-bifurcation regime depend on the form of the nonlinear feedback. For example, if a different kind of nonlinear feedback is used (e.g. $\delta x^{3}-\gamma x^{5}$ instead of $\left.-\delta x^{3}\right)$ in Eq. (1), then a different type of bifurcation (i.e. subcritical) may occur, as shown in Fig. 2.

Also, in general, the origin of the state space is a stable fixed point (equilibrium state) for most autonomous systems. Hence, in its simplest form, the key idea of the proposed method is to actively change the stability of the fixed point at the origin (by tuning the values of the controller parameters), and to design the nonlinear feedback such that a new stable fixed point or a limit cycle emerges close to the origin. These bifurcations, caused by the designed nonlinear feedback excitation for this simple scenario, are pitchfork or Hopf bifurcations. Next, changes in bifurcation boundaries (i.e. changes in the loci of bifurcation points) in the space of the controller parameters can be exploited to identify changes in structural parameters caused by damage. ${ }^{27}$ In this paper, in addition to the autonomous feedback, a time-dependent force is added to the nonlinear feedback excitation. The resulting system is unlike autonomous systems where pitchfork or Hopf bifurcations occur. Due to the explicit time-dependence of the excitation, the system exhibits more complex dynamics when the controller parameters are varied. Such qualitative or quantitative changes in dynamics are sensitive to very small changes in structural parameters. Therefore, observing the changes in bifurcation diagrams, and in particular the morphing of bifurcation boundaries caused by a nonlinear feedback combined with a time-dependent force is beneficial for detecting incipient damage. In comparison with pitchfork and Hopf bifurcation boundaries that can be analytically predicted by exploring the eigenvalues of the Jacobian 


\begin{tabular}{|l|c|c|c|}
\hline & (a) No mass is added & (b) $20 \mathrm{mg}$ mass is added & Relative Difference [\%] \\
\hline Mode 1 & 20.5589 & 20.4877 & 0.3460 \\
\hline Mode 2 & 123.2820 & 122.8804 & 0.3258 \\
\hline Mode 3 & 321.9955 & 321.0694 & 0.2876 \\
\hline Mode 4 & 563.9966 & 562.5962 & 0.2483 \\
\hline
\end{tabular}

Table 2. First 4 frequencies $(\mathrm{Hz})$ for the sensing beam with no mass, and with $20 \mathrm{mg}$ added at the tip.

matrix at the origin, ${ }^{27}$ investigating the changes in dynamics of the nonautonomous nonlinear system relies primarily on numerical analysis. Whether numerically simulating or experimentally implementing the proposed approach, the bifurcation diagrams and the locus of bifurcation points for detecting changes in structural parameters can be obtained by varying the values of the controller parameters in the numerical model or in the control circuit in the experimental setup.

\section{Experimental setup and procedure}

The sensing cantilever beam is composed of an aluminum alloy beam with two PZT patches symmetrically bonded on its surfaces near the clamp location using an epoxy adhesive, as shown in Fig. 1. The dimension and properties of the beam and PZT patches are given in Tab. 1. The two PZT patches have the same orientation of their polarization such that the bimorph configuration can generate larger deflections.

A laser vibrometer is used to measure the velocity at the tip of the beam. In addition to the laser vibrometer, there are several apparatus used for experiments including a function generator, an amplifier, a laptop, and a real-time processor. The real-time processor is used for data acquisition and signal processing, and can be controlled through a computer interface. Therefore, the controller circuits can be coded offline or online, and then uploaded from the host laptop to the real-time controller. The output analog signal is generated through the circuits, as a nonlinear function of the measured signal combined with a timedependent function. Meanwhile, the measured signal can be saved in a buffer of the real-time controller, and then downloaded to the host laptop for post-processing (such as creating bifurcation diagrams and tracking bifurcation points).

Overall, a nonlinear feedback excitation is formed by a feedback loop consisting of sending the analog signal measured from the laser vibrometer into the real-time processor, processing this signal via a designed circuit in the processor, amplifying the analog output signal from the processor, and finally applying the amplified voltage to the PZT patches to excite the beam. The net effect of the applied voltage is to introduce a torque at the ends of the piezo patches, causing the sensing beam to bend. The actual bifurcation point of the dynamics can be determined by varying the values of the parameters used in the controller and recording the values at which the dynamics changes qualitatively.

To explore the active bifurcation-based method for identifying parameter variations exploiting active bifurcation morphing, masses (made of wax) of different weights are attached to the sensing beam to create parameter variations in the system. The reason for using mass variations is that these variations are easily quantifiable, reproducible, and reversible. Furthermore, many applications use mass detectors, such as a class of biological and chemical sensors, and also certain corrosion detectors, which identify corrosion by detecting loss of mass in a structure.

\section{Finite element modeling}

The purpose of modeling the sensing beam is to develop a mathematical description for understanding and controlling the system behavior. The finite element method has been shown to be an effective tool for 

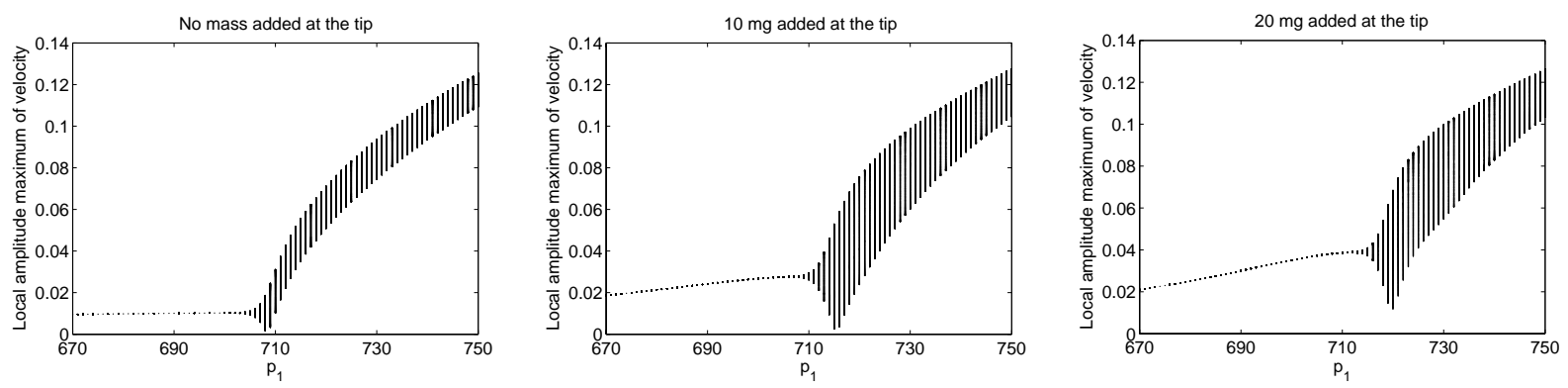

Figure 3. Bifurcation diagrams for the cases in which different amounts of mass (no mass, $10 \mathrm{mg}$, and $20 \mathrm{mg}$ ) are added at the tip of the beam.

modeling smart structures. ${ }^{30,33}$ In this study, the governing equation of the sensing beam is obtained using both regular beam elements and piezo beam elements (a composite beam composed of two piezoelectric layers perfectly bonded to a regular beam). Therefore, the mass and stiffness matrices of the sensing beam model include the contribution of the mass and stiffness of the PZT patches. The elemental mass and stiffness matrices are derived from the kinetic and strain energy equations of an element based on Euler-Bernoulli beam theory. Then, the global mass and stiffness matrices ( $\mathbf{M}$ and $\mathbf{K}$ ) are formed, and a Rayleigh damping matrix $\mathbf{C}$ is used in the modeling as

$$
\mathbf{C}=\alpha \mathbf{M}+\beta \mathbf{K}
$$

where $\alpha=2 \xi \omega_{1} \omega_{2} /\left(\omega_{1}+\omega_{2}\right), \beta=2 \xi /\left(\omega_{1}+\omega_{2}\right), \omega_{1}$ and $\omega_{2}$ are the first and second modal frequencies, and $\xi$ is the specified damping ratio. Also, based on the assumption of perfect bonding layer and effectively transferred shear force, ${ }^{28}$ the moment exerted at a node of a piezo beam element due to the applied voltage can be expressed as

$$
M_{0}=\frac{E_{b} t_{b} E_{p} t_{p}}{E_{b} t_{b}+6 E_{p} t_{p}} A_{b} \frac{d_{31} V}{t_{p}}
$$

where $E_{b}$ and $E_{p}$ are the Young's modulus of the aluminum beam and the PZT patches, $t_{b}$ and $t_{p}$ are the thickness of the aluminum beam and the PZT patches, $A_{b}$ is the cross section area of the aluminum beam, $d_{31}$ is the piezoelectric constant of the PZT patches, and $V$ is the applied voltage to the PZT patches. The applied voltage can be a combination of a nonlinear function of the measured signal and any time-dependent functions through a feedback loop. Thus, the nonlinear feedback excitation $\mathbf{F}$ can be modeled by assembling all (elemental) nodal forces, and using the specific form of the nonlinear control circuit (embedded in the real-time processor) and the gain of the amplifier. Finally, the equation of motion for the discretized sensing beam model subjected to the nonlinear feedback excitation can be expressed as

$$
\mathbf{M} \ddot{\mathbf{x}}+\mathbf{C} \dot{\mathbf{x}}+\mathbf{K x}=\mathbf{F},
$$

where $\mathbf{x}$ is the vector of nodal displacements. Eq. (4) can be expressed in state space representation by introducing a new vector $\mathbf{v}=\dot{\mathbf{x}}$ as follows

$$
\dot{\mathbf{X}}=\mathbf{A X}+\mathbf{B F}
$$

where

$$
\mathbf{A}=\left[\begin{array}{cc}
\mathbf{0} & \mathbf{I} \\
-\mathbf{M}^{-1} \mathbf{K} & -\mathbf{M}^{-1} \mathbf{C}
\end{array}\right], \mathbf{B}=\left[\begin{array}{c}
\mathbf{0} \\
\mathbf{M}^{-1}
\end{array}\right], \mathbf{X}=[\mathbf{x} \mathbf{v}]^{T}, \text { and } \mathbf{F}=\mathbf{G X}+\overline{\mathbf{F}}(\mathbf{X})+\overline{\mathbf{F}}(t) .
$$



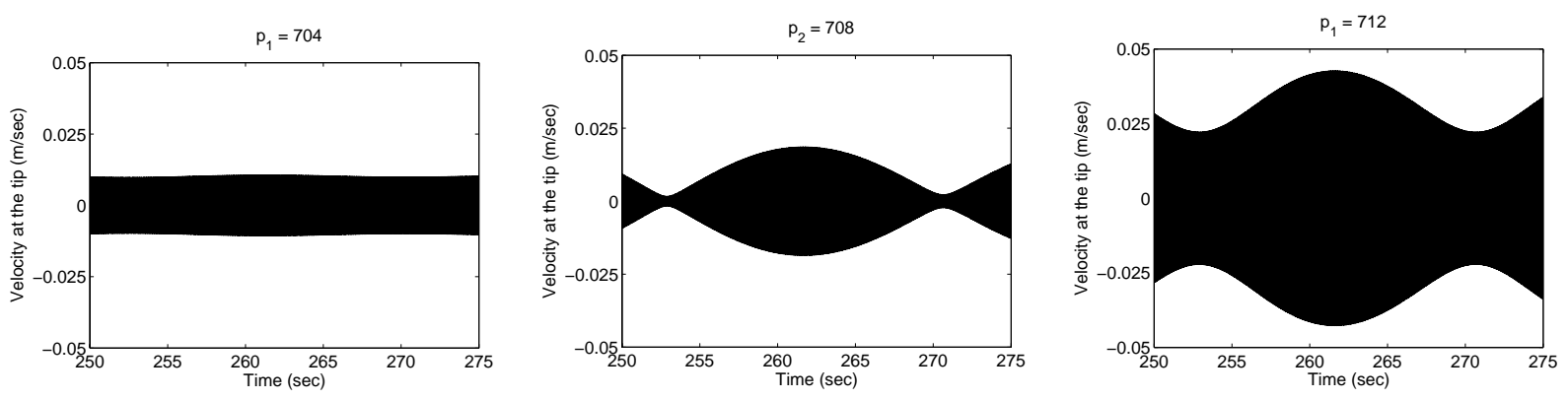

Figure 4. The time response of velocity at the tip of the sensing beam without added mass for different values of $p_{1}$ (i.e. 704,708 , and 712 ).

Herein, the nonlinear feedback excitation $\mathbf{F}$ is a function of $\mathbf{x}, \dot{\mathbf{x}}$, and $t$, and consists of nonlinear feedback $\overline{\mathbf{F}}(\mathbf{X})$, a time-dependent function $\overline{\mathbf{F}}(t)$, and linear feedback $\mathbf{G X}$, where $\mathbf{G}$ is a linear gain matrix. The dynamics of the sensing beam forced by nonlinear feedback excitation can be obtained by solving Eq. (5) using any time-marching scheme. To generate a bifurcation diagram computationally, the values of the controller parameters used in the nonlinear feedback excitation are varied, and the dynamics of the sensing beam is recorded from numerical integrations done for each set of parameters.

\section{Nonlinear feedback excitation design}

Two important questions in the experimental setup used herein are what combination of nonlinear functions of the measured signal, and what time-dependent functions should be utilized in the controller such that the bifurcation morphing due to small change in mass is sensitive. The design of the controller cannot be as simple as in most investigations using numerical simulation. As discussed in Section IV, the linear model of the sensing beam is obtained easily by the finite element method. In contrast, creating an appropriate form of nonlinear feedback excitation $\mathbf{F}$ is more challenging, especially experimentally.

The controller designed for the sensing beam draws inspiration from well-known driven dissipative flows. Specifically, the form of nonlinear feedback excitation can be defined by mimicking a driven van der Pol oscillator. To that aim, consider first that the cantilever beam may be approximated as a one degree-offreedom system (e.g. corresponding to the first Galerkin mode). The governing equation of a one degree-offreedom van der Pol oscillator can be expressed as

$$
\ddot{x}+x=b\left(1-x^{2}\right) \dot{x}+P \sin \Omega t,
$$

where $b, P$, and $\Omega$ are related to the controller parameters. This oscillator can exhibit diverse dynamics when different parameters are chosen. The main causes for the rich dynamics are nonlinearity and the harmonic force. However, the nonlinear form in Eq. (6) can not be directly used in nonlinear feedback excitation since the measured signal in our experiment is only the velocity of one point on the beam rather than both displacement and velocity. Therefore, Eq. (6) requires modifications. First, Eq. (6) can be integrated with respect to time to obtain

$$
\dot{x}+\int x d t=b x-\frac{b}{3} x^{3}-\frac{P}{\Omega} \cos \Omega t+c_{1},
$$

where $c_{1}$ is a constant of integration. Then, by introducing $y=\int x d t-c_{1}$, Eq. (7) can be written as

$$
\ddot{y}+\varepsilon \dot{y}+y=(b+\varepsilon) \dot{y}-\frac{b}{3} \dot{y}^{3}-\frac{P}{\Omega} \cos \Omega t,
$$

$$
6 \text { of } 10
$$



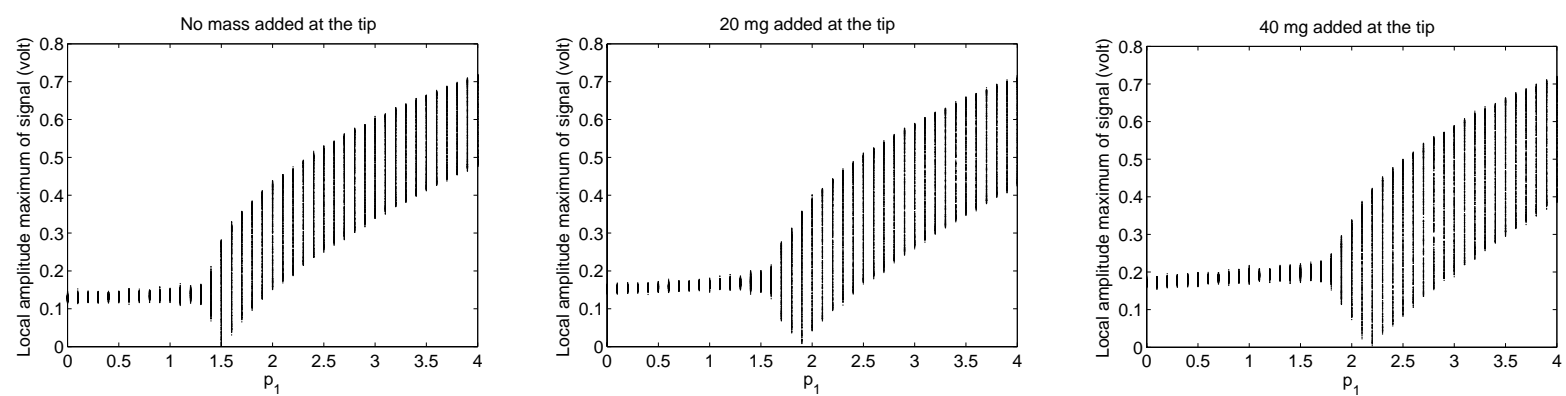

Figure 5. Bifurcation diagrams for the cases in which different amounts of mass (no mass, 20 mg, and $40 \mathrm{mg}$ ) are added at the tip of the beam, and the frequency of a harmonic function in the controller circuit is $18.8 \mathrm{~Hz}$.

where $\varepsilon$ characterizes the structural damping in the sensing beam. The dynamics of the new state $y$ is qualitatively similar to the one of the state $x$ in Eq. (6) if $x$ is smooth. Thus, $y$ can also exhibit various dynamics when different parameters are selected. In Eq. (8), the left hand side represents one mass-spring system, and the right hand side can be regarded as the nonlinear feedback excitation applied to the mass. One of the most important aspects of this design is that the nonlinear feedback excitation depends only on the velocity $\dot{y}$, and yet causes diverse dynamics. Based on this exploration, we specify the applied voltage $V$ in Eq. (3) as

$$
V=p_{1} v+p_{2} v^{3}+p_{3} \sin \Omega t
$$

where $v$ is the velocity measured using the laser vibrometer at the tip of the beam, and $p_{1}, p_{2}, p_{3}$, and $\Omega$ are the parameters of the nonlinear feedback excitation.

\section{Results}

Consider computational results first. Different parameter variations can be applied computationally, and in particular consider adding no mass, $10 \mathrm{mg}$, and $20 \mathrm{mg}$ at the tip of the sensing beam. Tab. 2 shows the first (lowest) four frequencies for the sensing beam with no mass and with $20 \mathrm{mg}$ mass added at the tip. The changes in these four frequencies due to a small amount of added mass is very small. Hence, most usual linear vibratory characteristics are not sensitive enough to this small change in mass. In contrast, the proposed approach takes advantage of nonlinearity resulting from the designed nonlinear feedback excitation. Herein, the values of the parameters $p_{2}, p_{3}$, and $\Omega$ are fixed and specified as 4000,1, and 128.81. The value of the parameter $p_{1}$ is varied from 670 to 750 with an increment of unity. The reason for choosing the parameter $p_{1}$ as the bifurcation parameter is to destabilize the original linear damping in the structure and create negative linear damping. In addition, the nonlinear damping (designed in the feedback excitation) allows the response of the structure to remain bounded, which is the same situation as for the driven van der Pol oscillator modeled in Eq. (8) (where $b<0$ ). Fig. 3 shows the bifurcation diagrams for the cases in which different amounts of mass (no mass, $10 \mathrm{mg}$, and $20 \mathrm{mg}$ ) are added at the tip of the beam. Compared to the low sensitivity of linear frequencies, the differences between the bifurcation diagrams are so significant that a very small amount of mass (such as $10 \mathrm{mg}$ ) can be easily detected. This enhanced sensitivity is the key feature for the proposed approach. Fig. 4 shows the time response of velocity at the tip of the beam without added mass for different values of $p_{1}$ (i.e. 704, 708, and 712). The three subplots in Fig. 4 represent three representative dynamics. The dynamics in the central subplot with the minimum local amplitude (similar to the beating phenomenon) can be considered as the transition from simple to complex limit cycles. Therefore, the corresponding value of the parameter may be regarded as a quantitative indicator for detecting changes in parameters. For example, in Fig. 3, the value of this particular point varies from 708 to 720 due to 

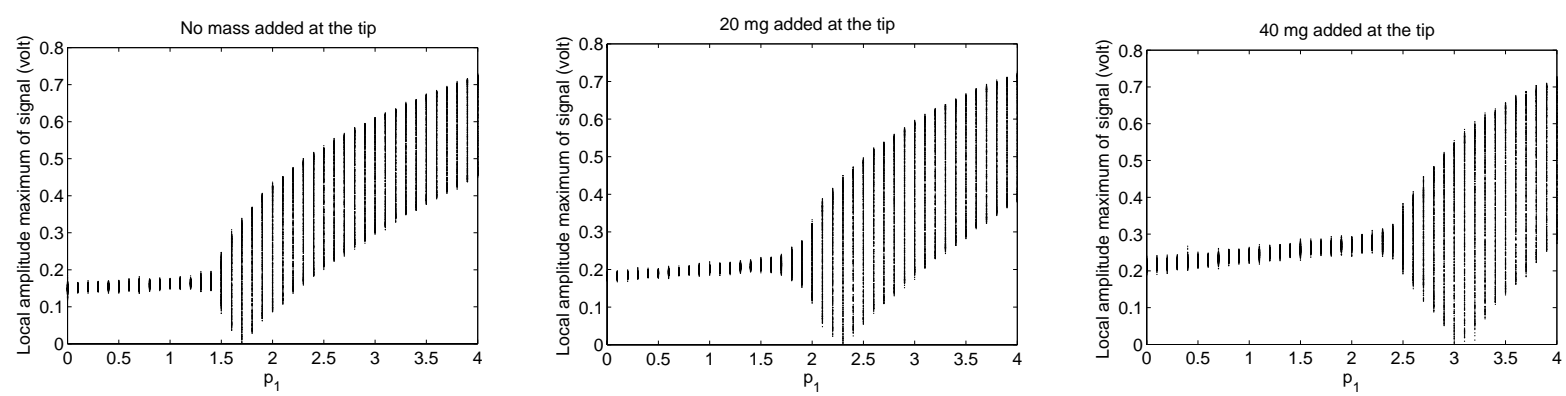

Figure 6. Bifurcation diagrams for the cases in which different amounts of mass (no mass, $20 \mathrm{mg}$, and $40 \mathrm{mg}$ ) are added at the tip of the beam, and the frequency of a harmonic function in the controller circuit is $18.9 \mathrm{~Hz}$.

different amounts of added mass.

Next, experimental results are discussed. Different amounts of mass (i.e. $10 \mathrm{mg}, 20 \mathrm{mg}$, and $40 \mathrm{mg}$ ) are added at the tip of the sensing beam to create variations in mass of the beam. Herein, the use of mass is due to the ease of removing it from and attaching it to the beam without changing the condition of the baseline case (i.e. no added mass). Then, the controller circuits embedded in the real-time processor can be programmed such that the output analog signal (voltage) has exactly the same form as Eq. (9).

Similar to numerical simulations, the parameter $p_{1}$ is varied, and the other parameters are fixed. Fig. 5 shows bifurcation diagrams resulting from experiments for the cases in which different amounts of mass (no mass, $20 \mathrm{mg}$, and $40 \mathrm{mg}$ ) are added at the tip of the beam, and the parameters $p_{2}, p_{3}$, and $\Omega$ are specified as 4000,1 , and $118.12(18.8 \mathrm{~Hz})$.

One may note that the morphing of the bifurcation diagram in Fig. 5 due to added mass is qualitatively similar to numerical results. For example, the value of the parameter $p_{1}$ corresponding to the dynamics with the minimum local amplitude shifts from left $\left(p_{1}=1.5\right)$ to right $\left(p_{1}=2.2\right)$. Also, for larger $p_{1}$ than each of these particular values, the width of the distribution of local amplitude maximum increases when the amount of mass increases.

Furthermore, we applied changes to the value of the frequency $\Omega$ of the harmonic function in the controller to explore its influences on the bifurcation morphing. Fig. 6 shows bifurcation diagrams obtained while the parameter $\Omega$ is specified as $118.75(18.9 \mathrm{~Hz})$. One may note that the bifurcation diagrams in Fig. 6 are more sensitive to variations in mass than the ones in Fig. 5. This is because the frequency used (of $18.9 \mathrm{~Hz}$ ) is closer to the fundamental frequency of the sensing beam. Note however, that the fundamental frequency cannot be approached too closely to gain higher sensitivity because the output signal surpasses the maximum output voltage limit of the hardware. Finally, the values of the parameters leading to the dynamics with the minimum local amplitude attract our attention since they can be considered as the transition points from simple to complex limit cycles. Therefore, we investigated the movement of these transition points (caused by added mass at the tip) in the parameter space spanned by $\Omega$ and $p_{1}$.

Figs. 7 and 8 show key results regarding the transition points in the parameter space for the cases where different amounts of mass (i.e. no mass, $10 \mathrm{mg}, 20 \mathrm{mg}$, and $40 \mathrm{mg}$ ) are added at the tip of the beam. The case where no mass is added is considered as baseline. The variations in the parameter $p_{1}$ with respect to the baseline due to different amounts of added mass for each specified frequency are shown in Fig. 8 .

From Fig. 8, one can notice that the variations in the parameter $p_{1}$ for the frequencies specified as $18.8 \mathrm{~Hz}$ and $18.9 \mathrm{~Hz}$ are nearly proportional to the small amount of added mass (e.g. $10 \mathrm{mg}$ and $20 \mathrm{mg}$ ). This proportionality provides an important basis for quantitatively predicting not only the presence of added mass, but also the amount of mass at the tip of the beam. Moreover, if the sensitivity for sensing the existence of added mass is more valued than the accuracy of predicting the amount of added mass, one may use a higher frequency (of $19 \mathrm{~Hz}$ ) to enhance sensitivity, as shown in Fig. 8. 


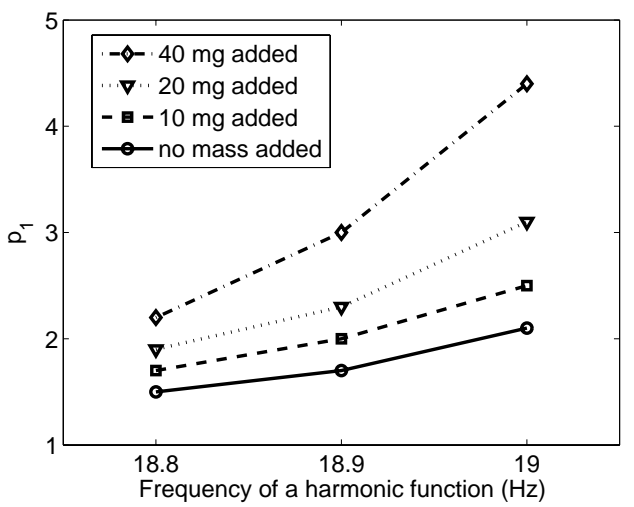

Figure 7. The transition points in the parameter space for the cases where different amounts of mass (no mass, $10 \mathrm{mg}, 20 \mathrm{mg}$, and $40 \mathrm{mg}$ ) are added at the tip of the beam.

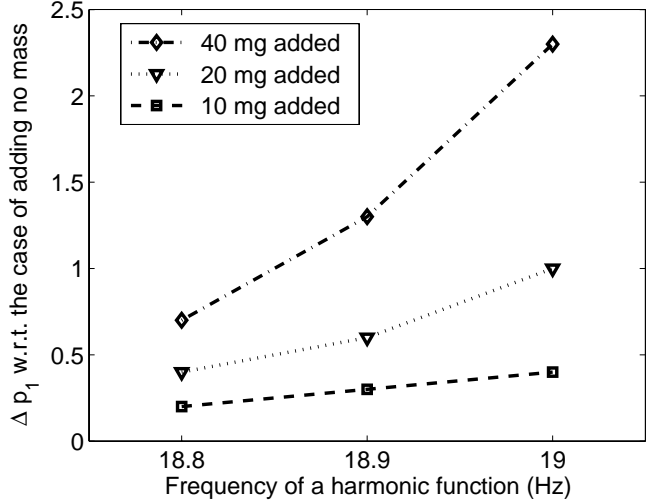

Figure 8. The difference in the parameter $p_{1}$ with respect to the baseline (no added mass) due to different amounts of added mass for each specified frequency.

\section{Conclusions}

A novel method for identifying parameter variations based on active bifurcation morphing has been demonstrated. To test this method experimentally, a smart cantilever sensing beam was built and excited by a nonlinear feedback excitation through two PZT patches bonded to the beam. The nonlinear feedback excitation was formed by a feedback loop which consists of sending the analog signal of velocity measured from a laser vibrometer into a real-time processor, using this signal in a designed circuit in the processor, amplifying the output signal from the processor, and finally applying the amplified voltage to the PZT patches. One of the key challenges in this study is how to design the nonlinear controller (i.e. the nonlinear feedback excitation) such that bifurcation morphing is very sensitive to small parametric variations. This problem was solved by employing a modified (driven) van der Pol oscillator, and testing the form of the nonlinear feedback excitation by numerical simulations based on a finite element model of the sensing beam. Both computational and experimental results showed that the sensitivity to small parametric variations (such as small changes in mass) can be significantly enhanced by the designed nonlinear feedback excitation. Also, the computational and experimental results show that the bifurcation morphing modes can be used as features for damage detection and sensing.

\section{References}

\footnotetext{
${ }^{1}$ Doebling, S. W., Farrar, C. R., Prime, M. B., and Shevitz, D. W., "Damage Identification and Health Monitoring of Structural and Mechanical Systems from Changes in Their Vibration Characteristics: A Literature Review," Report LA-13070MS, Los Alamos National Laboratories, Los Alamos, NM, 1996.

${ }^{2}$ Farrar, C. R., Doebling, S. W., and Nix, D. A., "Vibration-Based Structural Damage Identification," Philosophical Transactions of the Royal Society of London: A - Mathematical, Physical and Engineering Sciences, Vol. 359, No. 1778, 2001, pp. 131-149.

${ }^{3}$ Abdalla, M. O., Grigoriadis, K. M., and Zimmerman, D. C., "Enhanced Structural Damage Detection Using Alternating Projection Methods," AIAA Journal, Vol. 36, No. 7, 1998, pp. 1305-1311.

${ }^{4}$ Pappa, R. P., James, G. H., and Zimmerman, D. C., "Autonomous Modal Identification of the Space Shuttle Tail Rudder," Journal of Spacecraft and Rockets, Vol. 35, No. 2, 1998, pp. 163-169.

${ }^{5}$ Zimmerman, D. C., "Model Validation and Verification of Large and Complex Space Structures," Inverse Problems in Engineering, Vol. 8, No. 2, 2000, pp. 93-118.

${ }^{6}$ D'Souza, K. and Epureanu, B. I., "Damage Detection in Nonlinear Systems Using System Augmentation and Generalized Minimum Rank Perturbation Theory," Smart Materials and Structures, Vol. 14, No. 5, 2005, pp. 989-1000.
}

\section{9 of 10}


${ }^{7}$ Amizic, B., Amaravadi, V. K., Rao, V. S., and Derriso, M. M., "Two-Dimensional Wavelet Mapping Techniques for Damage Detection in Structural Systems," Proceedings of SPIE: Smart Structures and Materials 2002: Modeling, Signal Processing, and Control, edited by V. S. Rao, Vol. 4693, San Diego, California, 2002, pp. 267-278.

${ }^{8}$ Amaravadi, V. K., Mitchell, K., Rao, V. S., and Derriso, M. M., "Structural Integrity Monitoring of Composite Patch Repairs Using Wavelet Analysis and Neural Networks," Proceedings of SPIE: Smart Structures and Materials 2002: Smart Structures and Integrated Systems, edited by L. P. Davies, Vol. 4701, San Diego, California, 2002, pp. 156-166.

${ }^{9}$ Cao, T. T. and Zimmerman, D. C., "Procedure to Extract Ritz Vectors from Dynamic Testing Data," Journal of Structural Engineering, Vol. 125, No. 12, 1999, pp. 1393-1400.

${ }^{10}$ Zimmerman, D. C., "Looking into the Crystal Ball: The Continued Need for Multiple Viewpoints in Damage Detection," Key Engineering Materials: Damage Assessment of Structures, Vol. 167-168, No. 1, 1999, pp. 76-90.

${ }^{11}$ Ray, L. R. and Tian, L., "Damage detection in smart structures through sensitivity enhancing feedback control," Journal of Sound and Vibration, Vol. 227, No. 5, 1999, pp. 987-1002.

${ }^{12}$ Ray, L. R. and Koh, B. H., "Enhancing uniqueness properties in damage identification using sensitivity enhancing control," Materials Evaluation, Vol. 61, No. 10, 2003, pp. 1134-1142.

${ }^{13}$ Koh, B. H. and Ray, L. R., "Localisation of damage in smart structures through sensitivity enhancing feedback control," Mechanical Systems and Signal Processing, Vol. 17, No. 4, 2003, pp. 837-855.

${ }^{14}$ Kim, J. S., Wang, K. W., and Smith, E. C., "High-Authority Piezoelectric Actuation System Synthesis Through Mechanical Resonance and Electrical Tailoring," Journal of Intelligent Material Systems and Structures, Vol. 16, No. 1, 2005, pp. 21-31.

${ }^{15}$ Tang, J. and Wang, K. W., "Vibration confinement via optimal eigenvector assignment and piezoelectric networks," Journal of Vibration and Acoustics, Vol. 126, No. 1, 2004, pp. 27-36.

${ }^{16}$ Tang, J. and Wang, K. W., "Vibration delocalization of nearly periodic structures using coupled piezoelectric networks," Journal of Vibration and Acoustics, Vol. 125, No. 1, 2003, pp. 95-108.

${ }^{17}$ Nichols, J. M., Trickey, S. T., Todd, M. D., and Virgin, L. N., "Structural Health Monitoring Through Chaotic Interrogation," Meccanica, Vol. 38, No. 2, 2003, pp. 239-250.

${ }^{18}$ Nichols, J. M., Todd, M. D., and White, J. R., "Using State Space Predictive Modeling With Chaotic Interrogation in Detecting Joint Preload Loss in a Frame Structure Experiment," Smart Materials and Structures, Vol. 12, No. 2, 2003, pp. 580-601.

${ }^{19}$ Nichols, J. M., Todd, M. D., Seaver, M., and Virgin, L. N., "Use of Chaotic Excitation and Attractor Property Analysis in Structural Health Monitoring," Physical Review E, Vol. 67, No. 016209, 2003, pp. 1-8.

${ }^{20}$ Nichols, J. M., Virgin, L. N., Todd, M. D., and Nichols, J. D., "On the Use of Attractor Dimension as a Feature in Structural Health Monitoring," Mechanical Systems and Signal Processing, Vol. 17, No. 6, 2003, pp. 1305-1320.

${ }^{21}$ Trickey, S. T., Todd, M., Seaver, M., and Nichols, J., "Geometric Time Domain Methods of Vibration Based Damage Detection," Proceedings of the SPIE 9-th Smart Structures and Materials Conference, Vol. 1, San Diego, California, 2002, pp. $1-9$.

${ }^{22}$ Epureanu, B. I. and Yin, S. H., "Identification of Damage in an Aeroelastic System Based on Attractor Deformations," Computers and Structures, Vol. 82, No. 31-32, 2004, pp. 2743-2751.

${ }^{23}$ Epureanu, B. I., Tang, L. S., and Paidoussis, M. P., "Exploiting Chaotic Dynamics for Detecting Parametric Variations in Aeroelastic Systems," AIAA Journal, Vol. 42, No. 4, 2004, pp. 728-735.

${ }^{24}$ Epureanu, B. I., Yin, S. H., and Derriso, M. M., "Attractor-Based Damage Detection in a Plate Subjected to Supersonic Flows," Proceedings of SPIE 9-th International Symposium on NDE for Health Monitoring and Diagnostics, Vol. 5394, San Diego, California, 2004, pp. 340-350.

${ }^{25}$ Epureanu, B. I., Yin, S. H., and Derriso, M. M., "High-Sensitivity Damage Detection Based on Enhanced Nonlinear Dynamics," Smart Materials and Structures, Vol. 14, No. 2, 2005, pp. 321-327.

${ }^{26}$ Epureanu, B. I., Yin, S. H., and Dowell, E. H., "Enhanced Nonlinear Dynamics for Accurate Identification of Stiffness Loss in a Thermo-Shielding Panel," Nonlinear Dynamics, Vol. 39, No. 1-2, 2005, pp. 197-211.

${ }^{27}$ Yin, S. H. and Epureanu, B. I., "Enhanced Nonlinear Dynamics and Monitoring Bifurcation Morphing for the Identification of Parameter Variations," Journal of Fluids and Structures, 2005, to appear.

${ }^{28}$ Crawley, E. F. and Deluis, J., "Use of piezoelectric actuators as elements of intelligent structures," AIAA Journal, Vol. 25, No. 10, 1987, pp. 1373-1385.

${ }^{29}$ Hanagud, S., Obal, M. W., and Calise, A. J., "Optimal vibration control by the use of piezoceramic sensors and actuators," Journal of Guidance Control and Dynamics, Vol. 15, No. 5, 1992, pp. 1199-1206.

${ }^{30}$ Dosch, J., Leo, D., and Inman, D., "Modeling and control for vibration suppression of a flexible active structure," Journal of Guidance Control and Dynamics, Vol. 18, No. 2, 1995, pp. 340-346.

${ }^{31}$ Pai, P. F., Wen, B., Naser, A. S., and Schulz, M. J., "Structural vibration control using PZT patches and non-linear phenomena," Journal of Sound and Vibration, Vol. 215, No. 2, 1998, pp. 273-296.

${ }^{32}$ Schulz, M. J., Pai, P. F., and Inman, D. J., "Health monitoring and active control of composite structures using piezoceramic patches," Composites Part B-Engineering, Vol. 30, No. 7, 1999, pp. 713-725.

${ }^{33}$ Hwang, W. S. and Park, H. C., "Finite-element modeling of piezoelectric sensors and actuators," AIAA Journal, Vol. 31, No. 5, 1993, pp. 930-937. 